\title{
Speed-Accuracy Tradeoff in Trajectory-Based Tasks with Temporal Constraint
}

\author{
Xiaolei Zhou ${ }^{1}$, Xiang $\mathrm{Cao}^{2}$, and Xiangshi Ren ${ }^{1}$ \\ ${ }^{1}$ Kochi University of Technology, Kochi 782-8502, Japan \\ zxljapan@gmail.com, ren.xiangshi@kochi-tech.ac.jp \\ ${ }^{2}$ Microsoft Research Cambridge, Cambridge CB3 0FB, United Kingdom \\ xiangc@microsoft.com
}

\begin{abstract}
Speed-accuracy tradeoff is a common phenomenon in many types of human motor tasks. In general, the more accurately the task is to be accomplished, the more time it takes, and vice versa. In particular, when users attempt to complete the task with a specified amount of time, the accuracy of the task can be considered as a dependent variable to measure user performance. In this paper we investigate speed-accuracy tradeoff in trajectory-based tasks with temporal constraint, through a controlled experiment that manipulates the movement time $(M T)$ in addition to the tunnel amplitude $(A)$ and width $(W)$. A quantitative model is proposed and validated to predict the task accuracy in terms of lateral standard deviation $(S D)$ of the trajectory.
\end{abstract}

Keywords: Human performance model, speed-accuracy tradeoff, temporal constraint, trajectory-based tasks.

\section{Introduction}

An important research branch of human-computer interaction is to develop predictive models for human performance in fundamental interaction tasks. One of such tasks is the trajectory-based "steering" task, in which the user uses the input device such as a stylus to produce a trajectory ("stroke") through a "tunnel" with set amplitude (length) $A$ and width $W$. The movement time $(M T)$ of the steering tasks has been modeled by the steering law [1]: $M T=a+b(A / W)$, where $a$ and $b$ are empirically determined constants, and $A / W$ (index of difficulty or $I D$ ) characterizes the difficulty of the task. The steering law has been verified with several input devices [2], in different scales [3] and in simulated driving tasks [25].

The steering law models the relationship between the movement time of trajectorybased tasks and the task difficulty, determined by the tunnel amplitude $A$ and tunnel width $W$. In the steering law, the movement time $M T$ is the dependent variable. The more accurate the task is required (the narrower the tunnel width $W$ is), the longer the resulting movement time is. However, if we want to consider the opposite direction, i.e. inferring the actual trajectory accuracy given a specific movement time (or speed), the steering law does not enable us to make this prediction.

Given the bidirectional relationship between time and accuracy, it is worthwhile to establish a model that predicts the trajectory accuracy by considering the movement 
time as an independent variable. Such a model will supplement the steering law, and enrich our understanding of the speed-accuracy tradeoff in trajectory-based tasks. On the other hand, a prediction model of the trajectory accuracy also has practical implications. For example, pen gestures have been widely used to trigger commands. Such a model may allow us to estimate the deviation of the actual gesture stroke from the standard template at different drawing speeds, and improve the recognition and interpretation of the gestures. In a real world scenario, we may determine the optimal road width according to the marked driving speed.

Although speed-accuracy tradeoffs have been widely studied [14], [15], [17], [20], [23], these works have mostly focused on target acquisition tasks. In this paper, we sought to investigate the speed-accuracy tradeoff in trajectory-based tasks through a controlled experiment, and derive a quantitative model for predicting accuracy.

Previous studies on speed-accuracy tradeoffs have involved experimental protocols with two types of constraints: spatial constraint and temporal constraint, which differentiate the nature of the task. For example, in rapid aimed hand movements with spatial constraints, participants are required to move as quickly as possible to reach the target with width $W$ placed at distance $A$. The movement time is measured to reflect the task performance. This is a target acquisition task (also known as time-minimization task) and has been modeled by Fitts' law [6]. In rapid aimed hand movements with temporal constraints, participants are required to reach the target with a specified movement time. This is a paced reaching task (also known as a time-matching movement task) [20]. In this type of tasks, movement time is controlled and spatial variability of the movement is measured to reflect the accuracy. Similarly, in trajectory-based movements with spatial constraints, participants are required to produce a trajectory through a tunnel with length $A$ and width $W$ as quickly as possible. This is the standard steering task and has been modeled by the steering law [1]. However, if participants are required to produce a trajectory through a tunnel with length $A$ and width $W$ with a specified movement time, does regularity exist in the relationship between the trajectory accuracy and the task parameters? What kind of speed-accuracy tradeoff can be observed from trajectorybased task with temporal constraint? What are the differences between trajectory-based movements with temporal constraint and with spatial constraint? We sought to answer these questions in this paper.

\section{Related Work}

Depending on the stimulus of the task (target or trajectory) and the type of the constraint (spatial or temporal), research on the speed-accuracy tradeoffs can be divided into four categories as follows:

\subsection{Target-Based Tasks with Spatial Constraint}

One of the most famous and commonly used models in HCI is Fitts' law ${ }^{1}$ [6]. Fitts' law describes a logarithmic speed-accuracy tradeoff formulation in target acquisition

${ }^{1} M T=a+b \log _{2}(A / W)$, where $A$ is the amplitude of the movement, $W$ is the target width (i.e. the required accuracy), and $M T$ (the dependent variable) is the movement time taken to acquire the target. $a$ and $b$ are empirically determined constants. 
tasks with spatial constraint. Subsequently, the logarithmic speed accuracy tradeoff has been empirically validated for a wide variety of individual body joints [11], activities [11], and environmental conditions [8], [9]. Several variations of Fitts' law have also been presented by Welford [21] and Mackenzie [12].

Based on the traditional Fitts' law, Zhai et al. [26] investigated the speed-accuracy tradeoff based on participants' operational biases toward speed or accuracy, and attempted to derive a model incorporating both objective task parameters and subjective biases. However, a simple and linear model was not found by the empirical studies. Consequently, Ren et al. [19] established the SH-Model involving both the system and subjective factors based on the distribution of the actual movement time.

\subsection{Target-Based Tasks with Temporal Constraint}

Schmidt's law $^{2}$ [20] is closely related to Fitts' law. In their study, movement amplitude and time were manipulated, and the standard deviation of end points distribution was measured. Schmidt's law described a strong linear relationship between the movement speed and the standard deviation of the end points distribution.

In order to investigate the coexistence of spatial and temporal constraints in one motor task, Zelaznik et al. [24] manipulated movement time, amplitude and target width $(W>0)$ and discovered a similar linear speed-accuracy tradeoff. The target width did not affect the nature of the speed-accuracy tradeoff relationship.

In addition to researches that look at end point distribution, Wobbrock et al. [22] derived a predictive model for error rate through an experiment that manipulated target size, target distance and movement time. A logarithmic speed-accuracy tradeoff was found instead.

\subsection{Trajectory-Based Tasks with Spatial Constraint}

The steering law [1], described at the beginning of this paper, is the widely accepted model for trajectory-based tasks with spatial constraint. The movement time follows a linear relationship with the index of difficulty.

Subsequently, extensive researches have been done based on the steering law, such as models for steering through corners [16], steering within above-the-surface interaction layers using the tracking state of the stylus [7], and study of subjective biases toward speed or accuracy in steering tasks [27]. In addition, a pen stroke gesture model for predicting completion time of free hand trajectory drawing tasks has also been proposed [5].

\subsection{Trajectory-Based Tasks with Temporal Constraint}

So far, trajectory-based task with temporal constraint has not been investigated and modeled. Our aim in this paper is to investigate the trajectory accuracy when the movement time is considered as an independent variable in trajectory-based tasks,

${ }^{2} W_{e}=b(A / M T)$, where $W_{e}$ represents the standard deviation of end points, $A$ is the amplitude of the movement, and $M T$ (an independent variable) is the movement time as specified by the metronome. Therefore $A / M T$ characterizes the average movement speed. In this experiment, the target is a single line with zero width $(W=0)$. 
which will fill the void in human performance modeling research. In actual HCI applications, such a model may guide us to determine the optimal tunnel width given the speed requirements for particular trajectory-based interactions, such as navigating a hierarchical menu. Our model for trajectory accuracy may also have implications in scenarios beyond human-computer interaction, for example to determine optimal road widths for different driving speeds in traffic planning.

\section{Problem Definition and Hypothesis}

In this paper, we investigate the trajectory-based task of steering through a straight tunnel with temporal constraint (Fig.1). The user is required to complete the task with a specified movement time (within a tolerance range). Although the tunnel does have a finite width $W$ that the user supposedly stays within, this spatial constraint is not strictly enforced, i.e. the user may move outside the sides of the tunnel without failing the task.

We are interested in establishing a quantitative model for the trajectory accuracy described by the amount of lateral deviation throughout the trajectory produced. In practice this is represented by $S D$, the standard deviation of the y-coordinates (in the case of a horizontal tunnel) along the entire trajectory. The larger $S D$ is, the less accurate the trajectory is. Note that in contrast to the target acquisition task where accuracy is measured by the statistical distribution of a set of trials, here $S D$ describes the accuracy of a single steering movement trajectory.

In both Schmidt et al.'s study $(W=0)$ [20] and Zelaznik et al.'s study $(W>0)$ [24] on target acquisition tasks with temporal constraint, the standard deviation of the end point distribution is linearly related to the average movement speed. The effect of the target width on the accuracy was small, hence not included in their speed-accuracy tradeoff models. This might be explained as that in the target acquisition task, the target width only constrains the final corrective submovement but not the initial ballistic submovement (as discussed by Meyer et al. [13]). In contrast, in trajectory-based tasks the tunnel width constrains the entire movement, as the user is expected to produce a trajectory that stays within the tunnel all the time. Consequently, we hypothesize that in trajectory-based tasks with temporal constraint, not only is $S D$ related to the average movement speed $(A / M T)$, but also the tunnel width $W$ will have a considerable influence on $S D$. In order to provide a holistic understanding of all affecting factors, our speed-accuracy model for trajectory-based tasks should incorporate impacts of both factors. The correctness of this hypothesis will be verified through our experiment.

\section{Experiment}

\subsection{Apparatus}

The experiment was conducted on an IBM ThinkPad X41 Tablet PC with a 12.1-inch screen at the resolution of $1024 \times 768$, and a stylus as the input device. The experimental software was developed in Java. 


\subsection{Task}

The experiment used a basic trajectory-based task, which is steering through a horizontal straight tunnel with amplitude $A$ and width $W$ (Fig.1). The participant was required to move the stylus from the start line rightward to the end line through the tunnel, with a specified movement time (denoted as movement time goal or MT goal hereafter to distinguish from the actual movement time observed). A percentage temporal error tolerance parameter determined the acceptable range for the actual movement time. For example, if movement time goal was $200 \mathrm{~ms}$ and temporal error tolerance was $10 \%$, the actual movement time was allowed to range between $180 \mathrm{~ms}$ and $220 \mathrm{~ms}$ to be accepted. The participants were instructed that their movement time should be anywhere within the specified range.

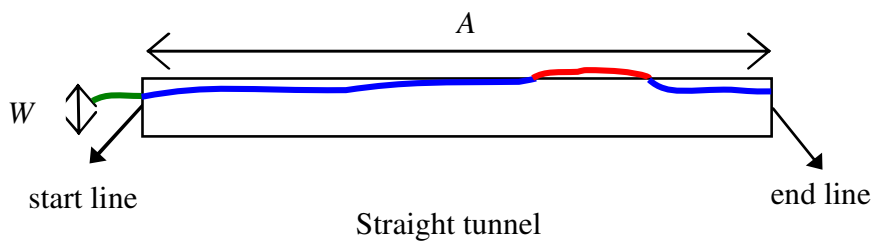

Fig. 1. Experimental task

Before the experiment began, the instructions were explained to the participants, who then conducted training trials until they fully understood the requirements and felt comfortable with the task. At the beginning of each trial, the tunnel to be steered was presented in black. After placing the stylus tip to the left of the start line, the subject began to move the stylus rightward. A green line was displayed to show the stylus trajectory produced by the participant. When the stylus crossed the start line, the trajectory line turned blue to signal that the task had begun. When the stylus crossed the end line, the task ended, and the actual movement time taken was displayed as feedback to the participant.

If the actual movement time was within the acceptable range, the trial was considered successful. Otherwise, the trial needed to be repeated until the actual movement time was within the acceptable range. For unsuccessful trials, the system indicated the percentage by which the trial was too fast or too slow, to help the participant adjust the movement time to meet the requirement.

Lifting the stylus between the start line and the end line was considered invalid and the trial needed to be repeated. The participant was instructed to try to keep the stylus within the upper and lower borders of the tunnel throughout the task. If the stylus was outside the tunnel borders during the trial, the trajectory part outside the borders was displayed in red as a warning (Fig.1), but the trial was not considered invalid.

\subsection{Measurements}

For each successful trial, the stylus position along the trajectory was sampled in intervals of $10 \mathrm{~ms}$. Based on these sample points, we calculated SD (Standard Deviation of 
y-coordinates of the sample points), and OPM (Out of Path Movement, percentage of sample points outside the tunnel) [10]. Calculated from the same set of data, both $S D$ and $O P M$ describe the accuracy of the trajectory, but from slightly different perspectives. $S D$ describes the original user behavior (lateral deviation) under the current stimuli, and provides understandings about the fundamental human capabilities; while $O P M$ evaluates how the user behavior satisfies the accuracy requirement (tunnel width) set by the particular task, and its implications are more on the user interaction side. For both $S D$ and $O P M$, higher values indicate lower accuracy.

In addition to the accuracy metrics, we recorded the actual movement time (or actual $M T$ ) for each successful trial to understand participants' performance on matching the MT goal. The actual MT is the time taken to move the stylus between the start line and the end line.

\subsection{Design and Procedure}

The experiment employed a mixed factorial design and combined within- and between-subject factors. The within-subject factors were $A(300,600,800$ pixels $), W$ (10, 25, 40, 55, 70 pixels), and MT goal (300, 500, 2000, 3500, 5000ms). The values of $M T$ goal were chosen according to the preliminary results of a pilot study, and values of $A$ and $W$ were chosen according to previous studies in [1] and [2].

The between-subject factor was temporal error tolerance $(10 \%, 20 \%$, and $40 \%)$. Zelaznik et al. [24] adopted 3 levels of temporal error tolerance to investigate the effect of temporal precision on the nature of the speed-accuracy tradeoff. To be consistent, in our experiment we also chose these 3 levels in order to investigate whether and how different levels of temporal error tolerance might affect the human performance and the nature of speed-accuracy tradeoff.

The participant was first briefed on the purpose of the experiment. Then 5 experiment sessions corresponding to the 5 MT goal conditions were tested in sequence. Within each session, the participant performed 3 successful trials for each combination of $A$ and $W$ respectively. Before each session began, the participant was informed of the current $M T$ goal and the relevant acceptable range of the actual movement time, and was allowed to perform practice trials until s/he felt comfortable.

The order of the MT goal conditions was counterbalanced using a Latin square pattern across participants. The order of the $A$ and $W$ conditions was randomized within each MT goal condition.

\subsection{Participants}

Thirty righted-handed people, aged from 21 to 34, participated in the experiment. They were assigned randomly to one of three temporal error tolerance groups $(10 \%$, $20 \%$ and $40 \%$ ), with 10 participants ( 8 males and 2 females) per group.

Therefore, the total number of successful trials performed was: 3 (trials) $\times 3$ (tunnel amplitude $A) \times 5$ (tunnel width $W) \times 5($ MT goal) $\times 3$ (temporal error tolerance group) $\times 10$ (participants per group $)=6,750$. 


\section{Results}

\subsection{Actual Movement Time (Actual $M T)$}

The actual movement time (actual MT) varied significantly with both the betweensubject factor temporal error tolerance $\left(F_{2,27}=8.97, p=.001\right)$, and all the withinsubject factors: $M T \operatorname{goal}\left(F_{4,108}=6584.77, p<.001\right), W\left(F_{4,108}=13.01, p<.001\right)$, and $A\left(F_{2,54}=249.24, p<.001\right)$. The mean actual $M T$ for the $10 \%, 20 \%$ and $40 \%$ temporal error tolerance groups were 2250, 2226 and 2089ms respectively. A significant interaction between temporal error tolerance and MT goal was observed on actual $M T\left(F_{8,108}=6.35, p<.001\right)$ (Fig.2). For $10 \%$ and $20 \%$ groups, the mean actual MT values approximated the MT goals. However, such was not the case for the $40 \%$ group. The mean actual MTs for the $300 \mathrm{~ms}$ to $5000 \mathrm{~ms}$ conditions were 312,495 , 1910, 3205, and 4522ms respectively. Post hoc pair-wise comparisons showed that actual MTs were almost equivalent with the MT goals for the $10 \%$ and $20 \%$ groups. However, for the $40 \%$ group, the actual MTs were equivalent with the other two groups only under the $300 \mathrm{~ms}$ and $500 \mathrm{~ms}$ condition, and significantly lower actual $M T$ s were observed than the other two groups under the 2000, 3500 and 5000ms conditions $(p<0.05)$.

Similar to the results obtained by Zelaznik [24], the results of $M T$ for the $40 \%$ group indicated a range effect [18]: longer-duration tasks exhibit an actual MT shorter than the $M T$ goal, indicating the participant moving at a more natural speed, faster than the speed dictated. The looser temporal constraint in the $40 \%$ group allowed this range effect to be observed, while the tighter constraints in the other two groups effectively eliminated the range effect.

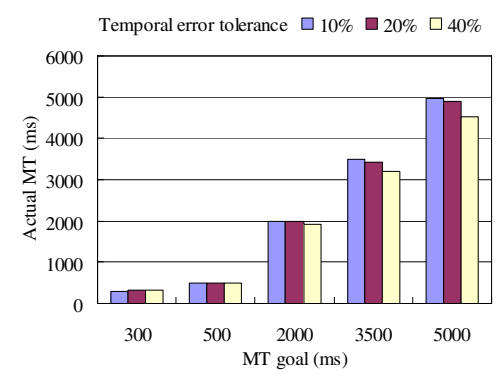

Fig. 2. Actual $M T$ vs. $M T$ goal for each temporal error tolerance

Another phenomenon was the significant interaction for temporal error tolerance $\times$ $W\left(F_{8,108}=4.36, p<.001\right)$, and temporal error tolerance $\times A\left(F_{4,54}=57.39, p<.001\right)$. In the $10 \%$ temporal error tolerance group, $W$ did not have a significant effect on actual $M T\left(F_{4,36}=1.68, p=.176\right)$. However, in both of the other two groups, $W$ had significant effects on actual $M T\left(F_{4,36}=4.91, p=.003\right.$ for $20 \%$ group; $F_{4,36}=8.49, p$ $<.001$ for $40 \%$ group), in that actual $M T$ decreased as $W$ increased. Similarly, the $10 \%$ group pair-wise comparisons revealed no significant difference in the actual $M T$ between the $A=600$ pixels and $A=800$ pixels conditions $(p=0.673)$. But in both the 
$20 \%$ and the $40 \%$ group, significant differences of actual MT were found among all three levels of $A$ ( $p=0.003)$, showing that actual $M T$ increased as $A$ increased. In the $20 \%$ and $40 \%$ groups the effects of $A$ and $W$ displayed the similar trends discovered by the steering law research [1], i.e., $M T$ increases with $A$ and decreases with $W$. Not surprisingly, because of the temporal constraints, the trends shown in our experiment were not strong enough to follow the linear relationship dictated by the steering law. Nevertheless, this is an interesting finding that even when people intentionally attempt to match a specific movement time, the underlying motor control mechanism still regulates the motion subconsciously within the allowable range and cannot be completely overridden. Again, in the $10 \%$ group, the strict temporal constraint prevented the trends from being observable.

\subsection{Trajectory Accuracy (SD)}

$S D$ measures the lateral deviation of the trajectory, as an indication of the trajectory accuracy. The grand mean of $S D$ was 2.85 pixels. $S D$ did not vary significantly with the between-subject factor temporal error tolerance $\left(F_{2,27}=1.36, p=.275\right)$, but varied significantly with all the within-subject factors: $M T$ goal $\left(F_{4,108}=121.04, p<\right.$ $.001), W\left(F_{4,108}=82.22, p<.001\right)$, and $A\left(F_{2,54}=292.42, p<.001\right) . S D$ decreased as $M T$ goal increased, showing that a longer movement time enabled participants to be more accurate. $S D$ increased as $W$ increased, showing that a wider tunnel allowed for less accurate movement. $S D$ also increased as $A$ increased, showing that a longer path (hence higher movement speed when other factors remain the same) resulted in less accurate movement. Table 1 summarizes these.

Table 1. Main effects on $S D$

\begin{tabular}{cccccc}
\hline$M T$ goal $(\mathrm{ms})$ & 300 & 500 & 2000 & 3500 & 5000 \\
\hline$S D$ (pixels) & 4.15 & 3.50 & 2.40 & 2.16 & 2.04 \\
\hline$W$ (pixels) & 10 & 25 & 40 & 55 & 70 \\
\hline$S D$ (pixels) & 2.33 & 2.50 & 2.86 & 3.19 & 3.38 \\
\hline$A$ (pixels) & & & & & \\
\hline$S D$ (pixels) & 300 & 600 & & 300 \\
\hline
\end{tabular}

Since no significant difference of $S D$ was observed among the three temporal error tolerance groups, we combined the data sets from the three groups in further analysis. No significant interaction for MT goal $\times W(p=.059)$ was observed on $S D$, as shown in Fig. 3 by the fact that the five regression lines are almost parallel, meaning that the effects of $M T$ goal and $W$ were independent. In addition, the correlations $\left(R^{2}\right)$ between $S D$ and $W$ are high $(0.875 \sim 0.985)$ for each $M T$ goal, showing that $S D$ follows a strong linear relationship with $W$ when other variables are factored out.

Similarly, no significant interaction between $W$ and $A$ were observed on $S D$, indicating that the effects of $W$ and $A$ were independent as well.

A significant interaction between $M T$ goal and $A\left(F_{8,72}=45.216, p<.001\right)$ was observed on $S D$ (Fig.4). The effect of $A$ increased as $M T$ goal decreased, as shown by the slopes of the regression lines. This is an intuitive observation if we consider the 


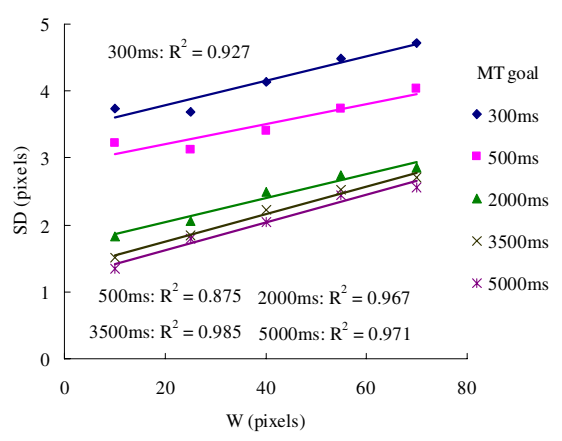

Fig. 3. Mean $S D$ vs. $W$ for each $M T$ goa.

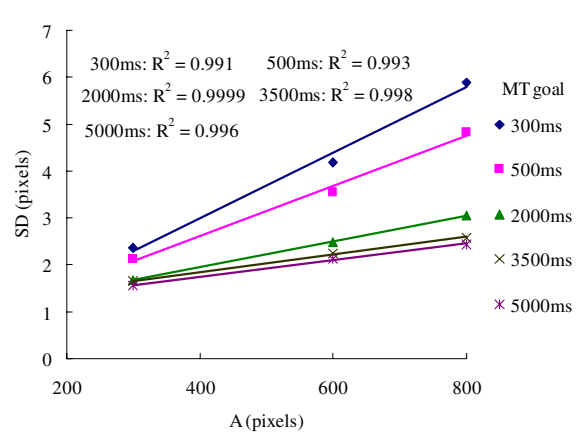

Fig. 4. Mean $S D$ vs. $A$ for each $M T$ goal

average movement speed that is $A / M T$. Smaller $M T$ goal resulted in larger changes on the anticipated movement speed for the same amount of change over $A$, and in turn larger changes on the movement accuracy. Similar to $W$, the correlations $\left(R^{2}\right)$ between $S D$ and $A$ are high for each $M T$ goal in Fig.4, showing that $S D$ follows a strong linear relationship with $A$ when other variables are factored out.

\subsection{Out of Path Movement (OPM)}

$O P M$ measures the percentage of the trajectory outside the tunnel, indicating how well the spatial constraint was satisfied. The grand mean of $O P M$ was $3.4 \%$. OPM did not vary significantly with the between-subject factor temporal error tolerance $\left(F_{2,27}\right.$ $=1.77, p=.189)$, but varied significantly with all within-subject factors: $M T$ goal $\left(F_{4}\right.$, $\left.{ }_{108}=148.53, p<.001\right), W\left(F_{4,108}=315.58, p<.001\right)$, and $A\left(F_{2,54}=128.88, p<.001\right)$. Table 2 summarizes mean $O P M$ under different conditions. Similar to $S D, O P M$ decreased as $M T$ goal increased, and increased as $A$ increased. However, different from $S D, O P M$ decreased as $W$ increased. It was easier for participants to keep the stylus inside a wider tunnel, despite that the produced trajectory itself becomes more relaxed (resulting in higher $S D$ ).

Table 2. Main effects on $O P M$

\begin{tabular}{|c|c|c|c|c|c|}
\hline$M T$ goal $(\mathrm{ms})$ & 300 & 500 & 2000 & 3500 & 5000 \\
\hline$O P M(\%)$ & 8.6 & 6.6 & 1.3 & 0.5 & 0.2 \\
\hline$W$ (pixels) & 10 & 25 & 40 & 55 & 70 \\
\hline$O P M(\%)$ & 14.3 & 2.3 & 4.0 & 0.2 & 0.1 \\
\hline$A$ (pixels) & \multicolumn{2}{|c|}{300} & 600 & \multicolumn{2}{|r|}{800} \\
\hline$O P M(\%)$ & \multicolumn{2}{|c|}{1.3} & 3.3 & \multicolumn{2}{|r|}{5.7} \\
\hline
\end{tabular}

Given that no significant difference of $O P M$ was observed among the three temporal error tolerance groups, we combined the data set from these three groups in further analysis on $O P M$. Significant interaction between $M T$ goal and $W\left(F_{16,144}=\right.$ 190.31, $p<.001$ ) was observed on $O P M$. The effect of $W$ increased as $M T$ goal 
decreased. As known from the analysis of $S D$, smaller $M T$ goal resulted in larger lateral deviation $(S D)$ in the trajectory, which contributed to the variety of $O P M$ values that depended heavily on the tunnel width. However when MT goal is larger, the resulting smaller $S D$ meant most of the trajectory would stay inside the tunnel, and in turn caused the uniformly small $O P M$. This finding is similar to the study results on subjective bias in steering tasks [27]. Significant interactions also exist in $A \times M T$ $\operatorname{goal}\left(F_{8,72}=35.31, p<.001\right)$ and $A \times W\left(F_{8,72}=115.86, p<.001\right)$.

\section{Model Deduction and Verification}

Based on the experimental results, we now attempt to establish a speed-accuracy tradeoff model that quantitatively predicts $S D$ from $A, W$ and $M T$ goal. Based on our analysis of $S D$, we concluded that:

- $S D$ is significantly affected by tunnel width $W$, tunnel amplitude $A$ and MT goal.

- $S D$ increases as $A$ and $W$ increase, and decreases as $M T$ goal increase.

- The relationship between $S D$ and $W$ is linear when other variables remain constant. Same for the relationship between $S D$ and $A$.

- The effects of $W$ and $M T$ goal on $S D$ are independent of each other (i.e. additive). Same for the effects of $W$ and $A$. The effects of $A$ and $M T$ goal on SD are not independent (i.e. not additive).

Considering all these properties, we speculated the following model to describe the speed-accuracy tradeoff in trajectory-based tasks with temporal constraint:

$$
S D=a+b W+c(A / M T) .
$$

where $W$ is the tunnel width, $A$ is the tunnel amplitude, $M T$ is the specified movement time (i.e. $M T$ goal), and $S D$ is the lateral standard deviation of the trajectory. $a, b$ and $c$ are empirically determined constants. $A / M T$ represents the average movement speed.

To verify the above model, we fit it to our experimental data using least-square regression. In addition to fitting to the entire data set, we also fit the model to the data from each temporal error tolerance group individually to test its performance under different conditions. Table 3 summarizes the regression coefficients and $R^{2}$ values.

Table 3. Regression results of the proposed model (1)

\begin{tabular}{ccccc}
\hline temporal error tolerance & $a$ & $b$ & $c$ & $R^{2}$ \\
\hline All & 1.08 & 0.0185 & 1.44 & 0.857 \\
$10 \%$ & 0.985 & 0.0209 & 1.20 & 0.800 \\
$20 \%$ & 1.25 & 0.0164 & 1.41 & 0.826 \\
$40 \%$ & 1.02 & 0.0181 & 1.71 & 0.880 \\
\hline
\end{tabular}

The model had a good fit with the entire data set $\left(R^{2}=0.857\right)$, as well as with data from all individual temporal error tolerance groups $\left(R^{2} \geq 0.800\right)$. This confirmed the validity of our model. 
This model also confirmed our initial hypothesis that in trajectory-based tasks with temporal constraint, $S D$ is not only related to the average movement speed $(A / M T)$, but also related to the tunnel width $W$. In order to further consolidate our model by comparing its performance with simpler alternatives, we tested an alternate model that ignored the effect of $W$ in model (1), i.e.:

$$
S D=a^{\prime}+b^{\prime}(A / M T) .
$$

Regression results showed that the $R^{2}$ values for model (2) are lower $\left(R^{2}=0.813\right.$ for $40 \%$ group, and $<0.77$ for all other conditions) than those of model (1) in all cases, therefore not considered a valid model. Detailed results are omitted given the space limitation. Unlike in target acquisition tasks, the effect of $W$ on $S D$ cannot be ignored in trajectory-based tasks. As such, we conclude that model (1) best describes the speed-accuracy tradeoff in trajectory-based tasks with temporal constraint.

\section{Discussion}

In our model, $S D$ measures the "average" accuracy throughout the entire trajectory. This is consistent with our original problem setup of a straight tunnel with uniform width $W$, and $A / M T$ is the "average" movement speed. However, if we consider the more general case in which both the tunnel width and the movement speed can vary throughout the trajectory, we could let $W_{P}$ and $V_{P}$ represent the local tunnel width and instant movement speed at a given point on the trajectory. As a result, $S D_{P}=a+b W_{P}$ $+c V_{P}$ might be used to predict the local expected lateral deviation at the point. This might help us design and analyze interactions using trajectories or tunnels of various shapes and properties, and understand them at a finer level.

Our experiment used a setup with relatively strict temporal constraint and nonstrict spatial constraint to investigate trajectory-based tasks and the degree that people can conform to this constraint (hence the metric $O P M$ ). Real-world trajectory-based tasks often have this non-strict spatial constraint property, such as tracing a drawing. Nevertheless, the visual stimulus of the spatial constraint affects the precision of the movement, as is reflected in the model. In our future investigation, we could naturally study the other variant where the spatial constraint is strictly enforced. Conversely, we may consider the case there is no explicit spatial constraint at all (i.e. tunnel width $W=0$ ), which essentially becomes a line tracing task. Fortunately, we might predict the user performance under this case by setting $W=0$ in our current model, which then becomes $S D=a+c(A / M T)$. This means the lateral deviation is linearly related to the movement speed only, a similar result to Schmidt's law [20]. Obviously, this prediction would need real experimental data to be validated.

Throughout this paper we have been referring to previous research on speedaccuracy tradeoff in target acquisition tasks as an analogy. However, we also want to emphasize the differences between trajectory-based tasks and target acquisition tasks, especially in terms of the notion of accuracy. In a target acquisition task, the movement accuracy is solely determined by the destination (end point) of the movement, for which we call the "destination accuracy". The spatial error in the destination is mainly caused by the ballistic nature of the movement, and is collinear to the movement. In contrast, in a trajectory-based task, the movement accuracy is determined by 
the entire process (trajectory) of the movement, for which we call the "process accuracy". The spatial error in the trajectory is mainly caused by the motor instability in the movement, and is perpendicular to the movement. These differences also contributed to the different forms of speed-accuracy tradeoff models for the two types of tasks. Similar comparisons can be made with other motor control tasks, For example, in a crossing task, destination accuracy and perpendicular errors coexist, which may result in yet another form of speed-accuracy tradeoff.

Since participants could not possibly finish a task with the specified movement time exactly, temporal error tolerance was introduced to define the range of acceptable movement time. Although our choice of testing multiple levels of temporal error tolerance did not result in observable effects on the trajectory accuracy, it did provide interesting observations on user behaviors in terms of the actual movement time taken. In particular, from the groups with higher temporal error tolerance values, we observed that the steering law as a fundamental motor control mechanism still affects the movement time, even when people consciously follow an explicit temporal requirement. We suspect that a similar effect might be present in other types of motor control tasks as well. This suggests that in time-critical applications, we can not overlook the inherent properties of the tasks and expect users to be able to perform at an arbitrary rate, even when accuracy is not the priority. On the other hand, we used a post hoc feedback mechanism about the user's temporal performance. How real-time feedback mechanisms (e.g. progressively filling the tunnel with color to indicate the elapsing of time) might affect the users' behaviors remains an interesting topic for further study.

\section{Conclusion and Future Work}

As the result of our investigation, we can now answer the questions we raised in the beginning: In trajectory-based tasks with temporal constraints, regularity does exist in the relationship between the trajectory accuracy and the task parameters, which is described by the speed-accuracy tradeoff model:

$$
S D=a+b W+c(A / M T)
$$

where $W$ is the tunnel width, $A$ is the tunnel amplitude, $M T$ is the specified movement time, and $S D$ represents the lateral standard deviation of the trajectory. $S D$ forms a linear relationship with both the tunnel width $W$ and average movement speed $(A / M T)$.

Regarding the comparison between temporally and spatially constrained trajectorybased movements, both of them reflect a linear speed-accuracy tradeoff. As investigated by [27], in spatially-constrained tasks with subjective biases, the lateral deviation of trajectory $(S D)$ is affected by the tunnel width $W$ and the subjective bias. In comparison, in temporally-constrained tasks the accuracy of trajectory $(S D)$ is affected by both the tunnel width $W$ and the average steering speed $(A / M T)$.

In the future, we plan to extend our investigation to trajectory-based tasks with zero tunnel width, non-uniform tunnel width, as well as trajectories of other shapes such as a circle. We also plan to test our model using other input devices, other forms of temporal feedback, or other reward-penalty mechanisms for the temporal constraint. 
In addition to spatial accuracy, we are interested in investigating the temporal accuracy, which describes human capabilities in matching the temporal constraints. Finally, we plan to investigate individual differences in terms of perception, estimation, and preference of the time constraints, especially for different age groups.

\section{References}

1. Accot, J., Zhai, S.: Beyond Fitts' Law: Models for Trajectory-Based HCI Tasks. In: Proc. CHI 1997, pp. 295-302. ACM Press, New York (1997)

2. Accot, J., Zhai, S.: Performance evaluation of input devices in trajectory-based tasks: an application of the steering law. In: Proc. CHI 1999, pp. 466-472. ACM Press, New York (1999)

3. Accot, J., Zhai, S.: Scale effects in steering law tasks. In: Proc. CHI 2001, pp. 1-8. ACM Press, New York (2001)

4. Annett, J., Golby, C.W., Kay, H.: The measurement of elements in an assembly task: The information output of the human motor system. Quarterly Journal of Experimental Psychology 10,1-11 (1958)

5. Cao, X., Zhai, S.: Modeling human performance of pen stroke gestures. In: Proc. CHI 2007, pp. 1495-1504. ACM Press, New York (2007)

6. Fitts, P.M.: The Information Capacity of the Human Motor System in Controlling the Amplitude of Movement. Journal of Experimental Psychology 47, 381-391 (1954)

7. Kattinakere, R.S., Grossman, T., Subramanian, S.: Modeling steering within above-thesurface interaction layers. In: Proc. CHI 2007, pp. 317-326. ACM Press, New York (2007)

8. Kerr, R.: Movement time in an underwater environment. Journal of Motor Behavior 5, 175-178 (1973)

9. Kerr, B.A., Langolf, G.D.: Speed of aiming movements. Quarterly Journal of Experimental Psychology 29, 475-481 (1977)

10. Kulikov, S., Mackenzie, I., Stuerzlinger, W.: Measuring the effective parameters of steering motions. In: Proc. CHI 2005, pp. 1569-1572. ACM Press, New York (2005)

11. Langolf, G.D., Chaffin, D.B., Foulke, J.A.: An investigation of Fitts' law using a wide range of movement amplitudes. Journal of Motor Behavior 8, 113-128 (1976)

12. MacKenzie, I.S.: A note on the information-theoretic basis for Fitts' law. Journal of Motor Behavior 21, 323-330 (1989)

13. Meyer, D.E., Abrams, R.A., Kornblum, S., Wright, C.E., Smith, J.E.K.: Optimality in human motor performance: Ideal control of rapid aimed movements. Psychological Review 95(3), 340-370 (1988)

14. Meyer, D.E., Smith, J.E.K., Kornblum, S., Abrams, R.A., Wright, C.E.: Speed-accuracy tradeoffs in aimed movements: Toward a theory of rapid voluntary action. In: Jeannerod, M. (ed.) Attention and Performance, vol. XIII, pp. 173-226. Lawrence Erlbaum, Hillsdale (1990)

15. Meyer, D.E., Smith, J.E.K., Wright, C.E.: Models for the speed and accuracy of aimed movements. Psychological Review 89(5), 449-482 (1982)

16. Pastel, R.: Measuring the difficulty of steering through corners. In: Proc. CHI 2006, pp. 1087-1096. ACM Press, New York (2006)

17. Plamondon, R., Alimi, A.M.: Speed/accuracy trade-offs in target-directed movements. Behavior and Brain Sciences 20(2), 279-349 (1997)

18. Poulton, E.C.: Range effects in experiments on people. American Journal of Psychology $88,3-32(1975)$ 
19. Ren, X., Kong, J., Jiang, Q.: SH-Model: A Model Based on both System and Human Effects for Pointing Task Evaluation. Journal of Information Processing Society of Japan (IPSJ) 46(5), 1343-1353 (2005)

20. Schmidt, R.A., Zelaznik, H., Hawkins, B., Frank, J.S., Quinn, J.T.: Motor-output variability: A theory for the accuracy of rapid motor acts. Psychological Review 86, 415-451 (1979)

21. Welford, A.T.: Fundamentals of skill. Methuen, London (1968)

22. Wobbrock, J.O., Cutrell, E., Harada, S., Mackenzie, I.S.: An error model for pointing based on Fitts' law. In: Proc. CHI 2008, pp. 1613-1622. ACM Press, New York (2008)

23. Wright, C.E., Meyer, D.E.: Conditions for a linear speed-accuracy trade-off in aimed movements. Quarterly Journal of Experimental Psychology 35A(2), 279-296 (1983)

24. Zelaznik, H.N., Mone, S., McCabe, G.P., Thaman, C.: Role of temporal and spatial precision in determining the nature of the speed-accuracy trade-off in aimed-hand movements. Journal of Experimental Psychology: Human Perception and Performance 14(2), 221-230 (1988)

25. Zhai, S., Accot, J., Woltjer, R.: Human Action Laws in Electronic Virtual Worlds - an empirical study of path steering performance in VR. Presence 13(2), 113-127 (2004)

26. Zhai, S., Kong, J., Ren, X.: Speed-accuracy tradeoff in Fitts' tasks - on the equivalency of actual and nominal pointing precision. International Journal of Human-Computer Studies 61(6), 823-856 (2004)

27. Zhou, X., Ren, X.: An Investigation of Subjective Operational Biases in Steering Tasks Evaluation. To appear in Behaviour \& Information Technology. Taylor \& Francis (in press) 